Article

\title{
A System-of-Systems Framework for Improved Human, Ecologic and Economic Well-Being
}

\author{
Ash M. Genaidy ${ }^{1, *}$, Ronald L. Huston ${ }^{2}$, Dionysios D. Dionysiou ${ }^{3}$ and Waldemar Karwowski ${ }^{4}$ \\ 1 Worldtek Inc., Cincinnati, OH 45249, USA \\ 2 Mechanical Engineering Program, University of Cincinnati, Cincinnati, OH 45221, USA; ron.huston@uc.edu \\ 3 Environmental Engineering \& Science Program, University of Cincinnati, Cincinnati, OH 45221, USA; \\ dionysdd@ucmail.uc.edu \\ 4 Department of Industrial Engineering, University of Central Florida, Orlando, FL 32816-2993, USA; \\ wkar@ucf.edu \\ * Correspondence: world_tek_inc@yahoo.com \\ Academic Editor: Yu-Pin Lin \\ Received: 12 February 2017; Accepted: 12 April 2017; Published: 15 April 2017
}

\begin{abstract}
Advances in technology and management not keeping pace with the ever-increasing urban problems" is attributed in this research to the poor understanding of person-focused governance of societal, environmental and economic entities. The objective of this paper is to present an adaptive institutional model of person-driven effectiveness and ineffectiveness. The model proposes that human, ecologic and economic outcomes are heavily influenced by a complex system of systems, spanning from individually unique "non-physical influencers" to a broader set of social and environmental influencers that have a common impact on the larger society-environment-economy (SEE) system. At the heart of the model is an analytic formulation that explains the phenomena of non-physical blocker, enhancer and indifferent, which are responsible for the adaptation and maladaptation of social agents and, accordingly, for the sustainability and unsustainability of SEE systems. Examples are provided to illustrate the model applications: (a) the non-physical and maladaptive syndromes as antecedents of multi-morbidity; and (b) the broadened and narrowed minds as sources of sustainability and unsustainability at the SEE system level within the context of emerging technologies such as engineered nanomaterials.
\end{abstract}

Keywords: sustainability; society; environment; technology

\section{Introduction}

In today's world and in the foreseeable future, we will be confronted with unprecedented and diverse global problems such as (a) increased world population; (b) rapid consumption of diminishing natural resources; (c) increased cost of healthcare accompanied by poorer health; and (d) increasing environmental pollution, all combined with growing urbanization in all parts of the world. Moreover, in the absence of major changes, these problems will become more and more acute and, if not seriously addressed, eventually they will transform into chronic events for those affected in human populations and ecosystems. At the same time, however, the world is becoming smaller in the sense that unprecedented advancements in communication technologies are connecting diverse parts of the world into a kind of small village, and emerging technologies are promising to solve the global problems. But there is a paradox, in that the profound technical advances cannot seem to be applied to effectively reducing the world's problems. This paradox has its roots in social, environmental, technical, and political issues that are currently converting local entities and communities into unsustainable systems—-systems that cannot keep pace with the basic and higher needs and wants of human populations and ecosystems. 
Other researchers have raised similar arguments from different perspectives [1-3]. Therefore, it has been suggested that it is time to launch a new dialogue on science and the environment with a focused effort on simultaneously improving human, ecologic and economic well-being [1]. Our thesis is that many of the aforementioned issues are due, in part, to our limited understanding for the person-focused architecture (PFA) and leader-driven functioning of the institutions comprising humans, communities, ecology, and economic and environmental entities. It is believed that, in addition to the physical-chemical factors impacting the diverse societies, environments and economies on the planet, intangible and intrinsic human factors play a major role in the aforementioned changes.

The research reported herein deals with the above subject, with the ultimate goal of finding solutions to the aforementioned diverse global problems simultaneously impacting environmental, social and economic entities. We present the phenomena of non-physical blocker (i.e., the negative forces in a person's inner milieu dominate the positive forces), enhancer (i.e., the positive forces in a person's inner milieu are ahead of the negative forces) and indifferent (i.e., both positive and negative forces in a person's inner milieu are in balance and hence neutralize each other) as major influencers of the adaptability and mal-adaptability of society-environment-economy (SEE) systems at both the individual and system levels. It is maintained in this research that sources of sustainability and un-sustainability are largely drawn from these phenomena due to their influence on human actions and inactions. With this in mind, we define sustainable systems as entities jointly leading to value-added benefits with minimal or no risks to all constituents of the larger SEE system within a defined time period.

\subsection{Overview of Prior Models}

This research is motivated by a multi-faceted diverse set of large-scale problems. Thus, we present examples of prior models below. One dominant early class of economic models has been concerned with rational behavior in economic decision-making. These models were set forth by the classic work of Simon [4-6] over several decades in attempts to correct the notion that "an economic man" is also a "rational" human. As the name implies, this class of models is deterministic in nature and relies on the rational component in a person-focused architecture or "cognitive controls" as termed in the psychological literature. We should point out that rational behavior, as will be elaborated in this research, is the product of not only the rational component, but also other components in the system. This issue was evaluated under the auspices of emotional controls as jointly interacting with cognitive controls, but it was not formally documented in this class of models [6]. Furthermore, the economic perspective should also integrate the societal and ecologic viewpoints.

A contemporary class of models characterizes human behavior in a given specific instance as a stochastic process. Here, the analysis is formulated in terms of an iterative solution of a set of governing equations [7-9]. This class of models assumes that people behave at a digital level, thus simplifying the complexity of human thought and consequently ignoring the innate personality and biases of individuals. To remedy these difficulties, researchers have developed increasingly complex models attempting to account for the complex structure of human thought $[10,11]$. These advanced models have been applied to human reaction to environmental and ecological issues [12,13].

Another class of contemporary psychological models has developed structural equations for the mind based on mental properties without accounting for its structural entities and their collective interactions [14]. Other psychological models have treated a group of individuals as a social network where each social agent is treated as a node of 0 or 1 without consideration for the intrinsic entities of the mental engine [15].

In medicine, human-mind interactions have been regarded as a given; yet, there are no models demonstrating the structural details of non-physical entities as well as links to the intrinsic causal factors with a call for further research into the subject $[16,17]$. Other attempts have been made, such as Iyer et al. [18] who established frameworks for meditation practices in India, without discussing entities of the mind responsible for achieving this ultimate state of peace and tranquility. 
It is our belief that factors influencing human behavior are central to the aforementioned paradox. Our thesis is that these influencing factors must be considered in order to transform unsustainable systems into sustainable ones. By examining the influencing factors of human behavior, we can address motivation leading to decision-making by institutional leaders. In our model, we use a dynamic structure of the components forming the basis for a person's reaction to his/her environment and the resulting decisions. To the best of our knowledge such an attempt has not been documented in the published literature.

\subsection{Study Goal}

The goal of our research is to model a person-focused system that describes the nonphysical influencing factors for individuals and their environmental interactions. These factors and interactions are manifested by a person's direction of action items. A person-focused "architecture" is defined as the underlying intrinsic basis for communication and interaction in urban communities and the larger SEE system. We explain the phenomena of non-physical blocker, enhancer and indifferent as the driving forces for the adaptability and non-adaptability of social agents and hence the influencers of SEE systems towards a path of sustainability or unsustainability.

We provide applications to illustrate the concepts from individual and SEE perspectives. The applications show the versatility of the model and its potential implications with respect to improving human, ecologic and economic well-being. It should be noted that the person-focused architecture is modeled as a system of systems as the person is powered by interactive systems connecting the non-physical, physical and social states as shown in Figure 1 [19].

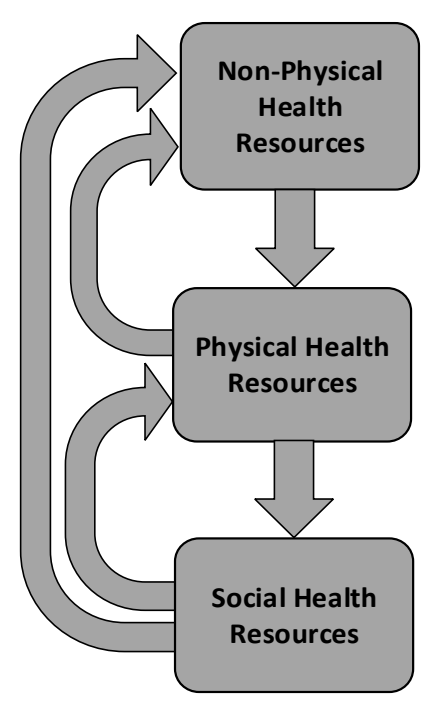

(a)

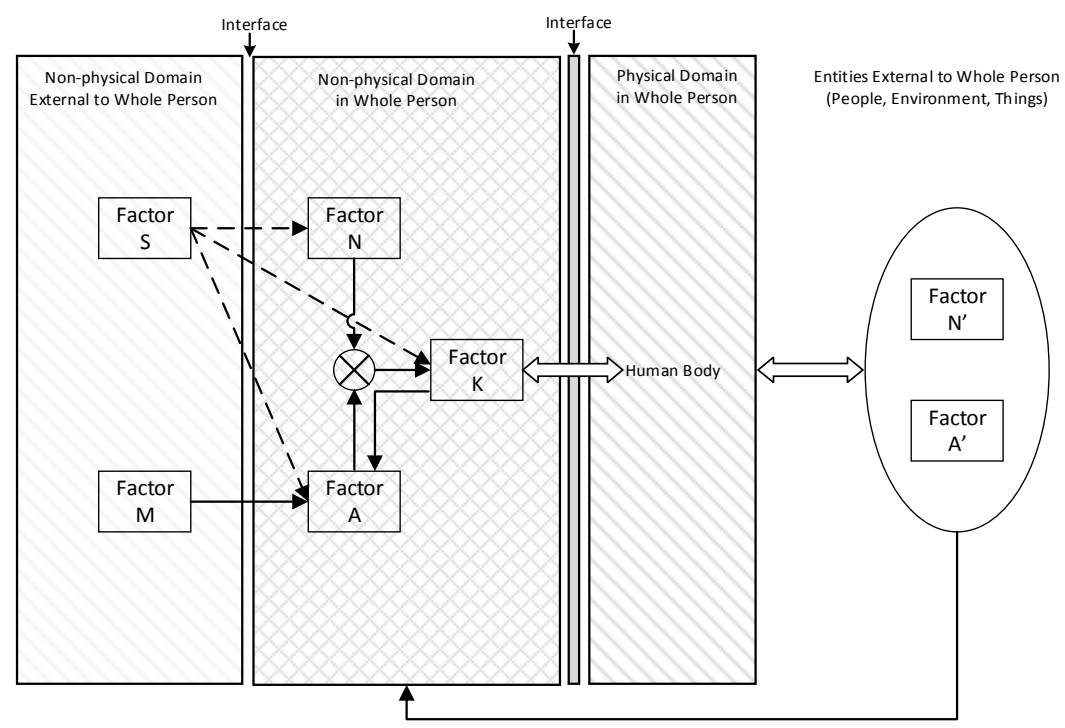

(b)

Figure 1. Person-focused system. (a) Interplay between non-physical, physical and social factors; (b) system of systems representation. 


\section{Person-Focused Architecture}

\subsection{Background}

The concepts of non-physical blocker, enhancer and indifferent can be traced back through the debate surrounding the mind-body dichotomy since the time of Descartes, due to the hidden (invisible) and intangible nature of the mind as opposed to the visible and tangible nature of the human body $[20,21]$. This debate has had great implications from a science standpoint.

The aforementioned paradox has led to the development of the biomedical model of medicine that focuses solely on biological and bodily factors; consequently, it does not take into account the "mind" factors [22,23]. In response, attempts have been made to account for the non-bodily factors in the biomedical model of medicine, such as the bio-psycho-social model, which unites the biological, psychological and social factors for treatment purposes [24]. Further attempts have considered uniting the mind and body as integral parts of living systems $[25,26]$.

Recently, Wade and Halligan [27] developed the biomedical model of illness in which people have two systems, with the first describing the whole self with dysfunction, termed impairment, and the second related to their organs with dysfunction, referred to as pathology. Although it is a good step forward towards accounting for non-bodily factors, it does not categorize the components of the non-physical entity. An editorial in Clinical Rehabilitation [28] echoes our appraisal, in that further research is warranted to advance our knowledge on the role of non-bodily factors in the biomedical model of medicine.

As already stated above and in the introduction, the structural entities of the mind have not been modeled as part of the biomedical model of medicine, and the mind factors have been accepted into practice as a possible explanation for the causal factors. Therefore, research is warranted to advance the theory of, among other things, body-mind interactions with respect to human illnesses and diseases.

An example is in order, here, to explain the implications of not considering the mind as an integral part of living systems from sustainability perspective. Consider two identical twins who have developed type II diabetes at about the same time, living in the same household and treated by the same physicians (i.e., primary care physician and/or specialists). With all these similarities, however, one of the twins is affected with an additional number of acute complications compared to the other. A question may then arise as to why the cost of care of one sibling is double relative to the other (assuming everything is the same). The obvious answer is the non-bodily factors that greatly influence lifestyle factors-and hence the body_-in feed-forward and feedback fashion as depicted in Figure 1a. In this diagram, social entities are added in order to provide the minimum structure for person-focused functioning in a larger system and generalize the views of mind-body entities at an SEE level. All in all, if these factors are not accounted for, the US healthcare system will remain on a path toward unsustainability $[29,30]$.

To address these issues, we built the PFA architecture based upon research in the scientific, philosophical, and even spiritual literature. Specifically, the components of the PFA and their interrelationships were initially inspired by accounts in ancient writings (including those in the Bible and the Koran) together with the smart logic of common sense, civil discourse, and conventional wisdom. From this broad background, the PFA was built upon the acquired knowledge and experience of the authors in engineering, human factors, and environmental sciences, including earlier writings of the authors [31,32].

\subsection{Model Description}

The person-focused architecture (PFA) of an individual is discussed with reference to a "community" consisting of people and other living beings (e.g., animals and birds in an ecosystem) and non-living entities (e.g., buildings), some of which are visible and obvious to an individual and others which may be less obvious and intangible but still have significant influence on the person (e.g., the health of an individual is influenced by the health of those with whom he/she interacts). An analogy 
may be gravity: it can't be seen but it can be measured, hence, one can determine its influence on physical items. Other less obvious examples may be unknown and undetectable pollutants, high frequency vibrations, and background noise.

The PFA Model maintains that a person consists of two layers: physical or outer (i.e., visible or human body, physical environment, etc.), and nonphysical or inner (i.e., "hidden" such as developmental, cellular, chromosomal, etc.). We know much about the physical human body via scientific discoveries because it is obviously tangible. On the other hand, we have a limited understanding of the complex interactions of the many nonphysical influencers, some of which are less tangible (e.g., how does childhood stress result in permanent chromosomal telomere changes which then influence a person's health for their entire life?).

The internal complex system of systems (SOS) is a collection of collaborative systems bonded together under defined rules and regulations with the goal of exhibiting the complex properties of living systems [33-35]. In this context, each individual has a component structure of "1" or "0" decision nodes that lead to action/inaction, which in turn exerts its resultant influence on the overall SOS. An imbalance in these influencers can push one toward poorer or better human outcomes. For purposes of this discussion, this internal SOS is described in general terms in order to then describe the structure and function use and relate it in the next section to examples on human and ecological health.

The inner layer of PFA consists of three components, (a) factor " $\mathrm{A}$ "; (b) factor " $\mathrm{N}$ "; and (c) factor " $K$ " (Figure 1b) and makes up the non-physical resources for the person. These three components are influenced by two external invisible inputs, factor " $\mathrm{S}$ " and factor " $\mathrm{M}$ ", with unipolar forces (i.e., one force is only negative " $\mathrm{S}$ ", and the other is purely positive " $\mathrm{M}$ "). The net output of the interactions of these factors is what we believe drives individual actions/inactions resulting in net human outcomes (e.g., overall health status both physical and mental). A brief definition is given below for each component followed by a discussion of their roles and their relationships in the person-focused architecture:

(a) Factor $\mathrm{A}$ is a rational component that is powered by the voice of reason and logic. It is modeled as a bipolar force (i.e., cognitive force), assuming that the power of logic and reason is modeled as a positive phenomenon and the power of illogic and unreason is regarded as a negative phenomenon.

(b) Factor $\mathrm{N}$ is the source of energy for the person, but with limited logic and reason capabilities (i.e., impulsive energy). This source of energy can be positive (i.e., enhancing the person's capability) or negative (i.e., diminishing the person's capability).

(c) Factors $\mathrm{A}$ and $\mathrm{N}$ are in constant interaction with the resultant value providing an input to factor $\mathrm{K}$.

(d) Factor $\mathrm{K}$ is the recipient and integrator of information from the internal milieu via factors $\mathrm{A}$ and $\mathrm{N}$ as well as factors $\mathrm{S}$ and $\mathrm{M}$ (see below). Structurally, it combines attributes of factors $\mathrm{A}$ and $\mathrm{N}$ for post-processing purposes. In addition, Factor K has extra sensory capabilities connecting it with the external environment for receipt of information input via two mechanisms: (1) directly via the channels of communication of the body (e.g., visual, auditory); and (2) indirectly by other means unknown to us. Information from the external environment is passed via factor $\mathrm{K}$ without any further processing to factor A for storage in its internal memory. This information is also temporarily stored in the memory of factor $\mathrm{K}$ for later usage in a decision making process in a given situation. Collectively, factor $\mathrm{K}$ renders the final decision on the basis of information from the internal and external milieus for action item orders to the outer layer. After it is made, information on the final decision is passed to factor A where it is stored in the long-term memory, residing as part of its cumulative experience amassed over the course of diverse situations and scenarios. (A note of clarification is warranted on the unknown route of information input to factor $\mathrm{K}$. This mechanism can be activated for example in extreme circumstances such as emergency situations or near death experiences). 
(e) Factor $\mathrm{M}$ is a positive phenomenon and provides consultative reason and logic to factor $\mathrm{A}$ via a one-to-one relationship. This unipolar positive phenomenon also has direct input to factor $\mathrm{K}$, particularly at times when the internal milieu is in a great state of turbulence.

(f) Factor $\mathrm{S}$ is a negative complex phenomenon and interacts with factors $\mathrm{N}, \mathrm{A}$ and $\mathrm{K}$ via a one-to-many relationship. Its goal is to augment the power of unreason and to diminish the power of reason via continuous monitoring of the inner layer in the PFA.

The state of factor $\mathrm{N}$ (i.e., output) can be described in terms of two-dimensional variables, that is, level of activation and energy type. Factor $\mathrm{N}$ may reside in one of four general sources of energy: (1) high-positive; (2) low-positive; (3) high-negative; and (4) low-negative. A person may reside anywhere in the spectrum of those four general states, with his/her position determined by the relative balance of the influencing factors (Figure 2a). An example of a high-positive energy source is hope (or optimism). This state is a major source of personal energy. Alternatively, despair (or pessimism) is a low state of negative energy source that is the opposite of hope and acts as a barrier for constructive human actions, often by leading to inaction. The low-positive energy source, such as peace and tranquility, leads to steady human actions in the most efficient and effective ways. Contrary to that, a high-energy negative source, such as anger and distress, results in a suboptimal source of energy, though it may be an essential component for survivability.

The state of factor A can also be described in terms of two parameters: (a) thinking mode-positive and negative; and (b) force magnitude-low and high (Figure $2 b$ ). Both parameters are influenced by stored personal experiences, interaction with factor $\mathrm{N}$, and inputs from factors $\mathrm{S}$ and $\mathrm{M}$. At birth, the default value for factor $\mathrm{A}$ is positioned in the positive domain. As people get older, they navigate across the full spectrum of positive and negative thinking with the resultant at any given time depending on the aforementioned influencers. Of particular interest here is the interaction between factors $\mathrm{A}$ and $\mathrm{N}$.

When factor $\mathrm{A}$ is in a state of positive thinking and factor $\mathrm{N}$ is in a state of positive energy, the role of factor $\mathrm{N}$ can be regarded as an amplifier for the positive thinking mode of factor $\mathrm{A}$. In this instance, the power of positive irrationality has a major impact on human actions allowing a person to behave in an exhilarated style. We maintain, here, that rational productive behavior is the product of the positive thinking mode of factor $\mathrm{A}$ and the positive rational mode of factor N. For example, consider a salesperson with great enthusiasm and belief in his/her product and with a high energy level. This is likely to lead to high sales. Alternatively, when factor $\mathrm{N}$ behaves as a negative energy source, it can act as a de-amplifier to factor A and steer it toward a state of negative thinking, if it can exceed the level of positive thinking for factor A. As expected, this will lead to irrational unproductive human behavior. If the above salesperson has doubts about the value or quality of his/her product and is tired with low energy level, sales are likely to be low.

When operating in a negative thinking mode, factor A is more likely than not synchronized with the negative domain of factor $N$. Under these circumstances, the negative thinking process is operating in a destructive (i.e., negative) mode from a base of negative experiences. In a "low" negative thinking mode, the person acts in a suboptimal fashion, but yet is still functional. "High" negative thinking makes the person move toward the path of negative irrational behavior that is detrimental to the community. An example is a disgruntled employee.

To enhance the negative phenomenon in the internal milieu of a person, factor $\mathrm{S}$ provides ideas and support on a constant basis to factor $\mathrm{N}$ in order to influence factor $\mathrm{A}$ towards the mode of negative thinking. Because of its destructive nature, factor $\mathrm{S}$ is capable of creating a barrage of negative concepts over time in the internal milieu. That is, it can be modeled as a train of negative impulses during the course of time, with each impulse being short lived and different from others in the train. Factor $\mathrm{N}$, on the other hand, may persist with one negative thought and promotes it on a continuous basis; therefore, it can be modeled as a finite step function simulating the "all" or "nothing" approach in a given situation. 


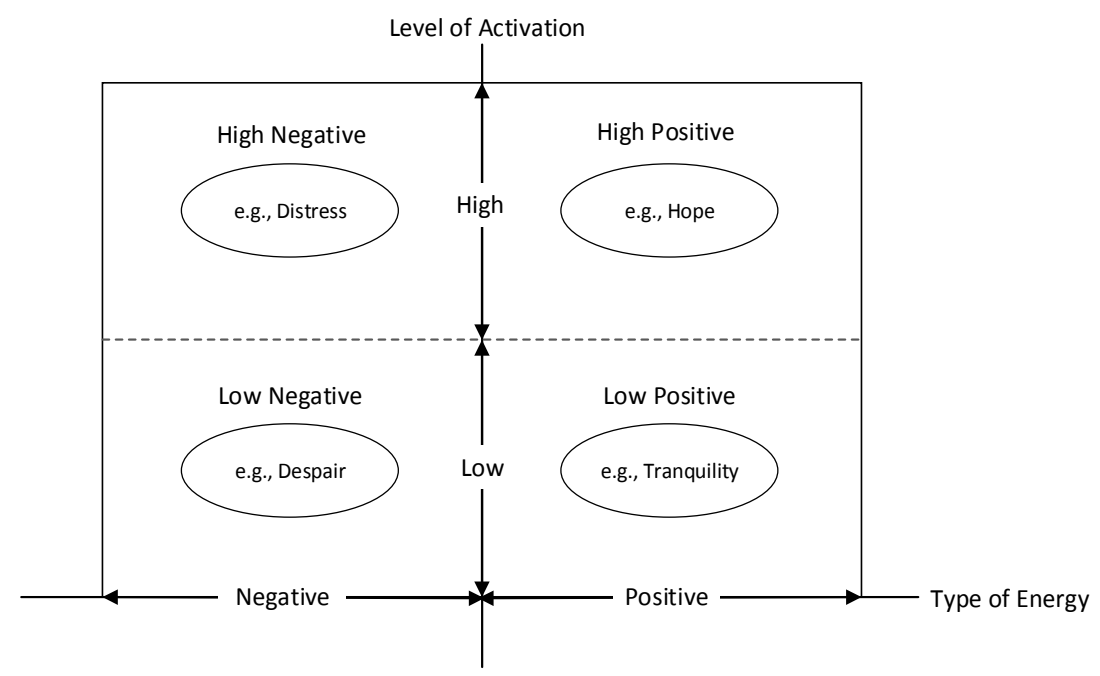

(a)

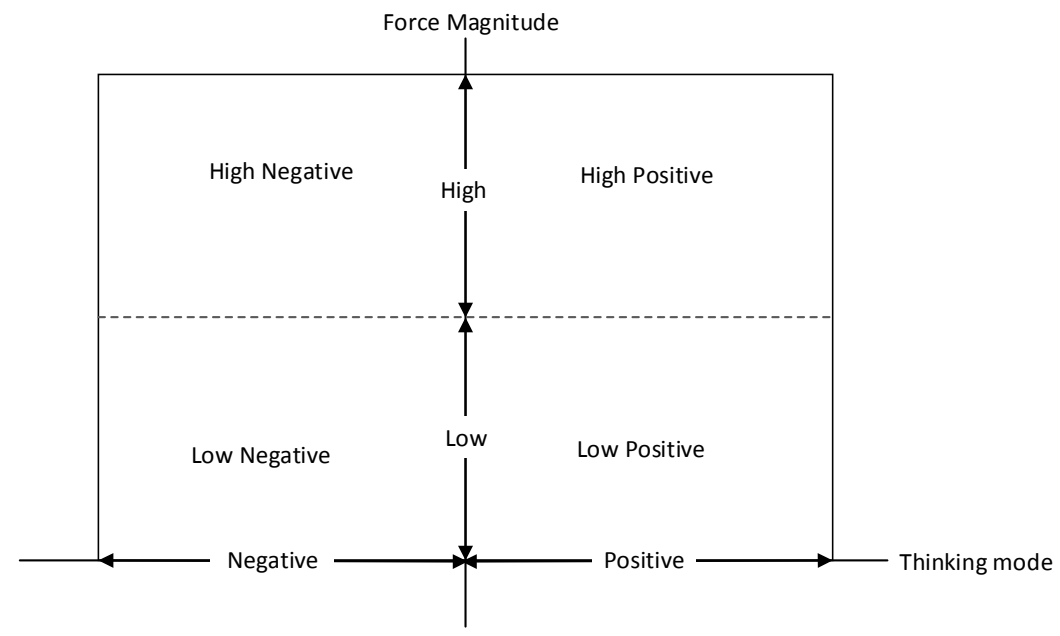

(b)

Figure 2. States of factors A and N. (a) States of factor N; (b) states of factor A.

The role of factor $\mathrm{S}$ in influencing factor $\mathrm{A}$ is focused on limiting its functional capabilities via two routes: (1) distracting and confusing the positive thinking capabilities; and (2) enhancing the negative thinking model. Inhibiting the positive thinking capabilities of factor A may be accomplished in several ways. The first way is via a "false" positive signal that attempts to portray the negative phenomena in life as a pseudo-positive experience to factor A to promote the performance of such actions. Another way is via a "false" negative signal. An example might be to paint a picture of a person who contributes to charity as becoming poor if he/she continues to do good deeds to others or perform actions that may benefit nature at large. A third way is to block the memory channels for the positive thinking mode; consequently, factor A cannot carry out its own function. To enhance the negative thinking capability for factor $\mathrm{A}$, factor $\mathrm{S}$ works on replacing the stored experience for making good decisions with false information leading to bad decisions. It can additionally act to disturb the existing stored experiences.

In view of the above, a person must be able to distinguish between the "true" and "false" signals obtained by the internal milieu to make rational and positive decisions. The false signal is indeed inputted as discussed from factor $\mathrm{S}$ and the "true" signal is obtained from factor M as well as the indirect mechanism unknown to us. In this regard one may, for example, be able to distinguish it as 
follows: if it is too good to be true or it is too depressing, then the signal stems from factor S, otherwise it emanates from factor $\mathrm{M}$ or the indirect mechanism unknown to us.

Finally, the components of the internal milieu inside the person may be influenced by feedback from other persons in a community (e.g., factors $\mathrm{A}^{\prime}$ and $\mathrm{N}^{\prime}$ in Figure $1 \mathrm{~b}$ ). The roles of $\mathrm{A}^{\prime}$ and $\mathrm{N}^{\prime}$ exemplified in other people who come in contact with the person can have a major impact on the decision-making capability of the person. That is, there are two types of feedback signals that are channeled to factors $\mathrm{A}$ and $\mathrm{N}$ of a person. The positive ideas are enhanced via the rational component of factor A and the irrational positive mode of factor N. On the other hand, the negative ideas are fed to the negative thinking mode of factor $\mathrm{A}$ and the irrational negative mode of factor $\mathrm{N}$. This may be best exemplified by the classic example of the advice of good parents to their kids to avoid hanging around "bad kids" and to stick to "good" ones. Therefore, one may need to exercise caution with respect to the "true" and "false" signals and cues received from others.

\subsection{Supporting Evidence for Model Components}

Factors $\mathrm{A}$ and $\mathrm{N}$ are consistent with the psychological literature on cognitive and emotional resources that has been available for many years [36-39].

Factors M and S are analogous to "good" and "evil" in the philosophical sense. Behind these characterizations, however, are external entities, albeit without tangible external manifestation, and are still frequently accepted internally.

Alternatively, factor $\mathrm{K}$ has no explicit psychological nor philosophical bias. Nevertheless, in everyday conversation, but without firm evidence, factor $\mathrm{K}$ is regarded like a person with a "good heart" or like a "wicked person". Indirect evidence suggests that collective psychological processes characterizing the biopsychosocial model during active goal pursuit may indeed be instrumental in influencing cardiovascular responses [40]. That is, the integrated mental engine has a connection to the physical heart without clear evidence about the causal relationships.

\subsection{Analytic Perspective of Component Interactions}

The influence of a person's internal milieu on his/her external environment can be sensed in terms of his/her cognitive and emotional enhancement or block, which is reflected in his/her (a) human behavior that acts as the mediating factor between the inner and outer layers; and (b) physiological functioning throughput. As such, the good and the ill of the inner milieu of the person may be represented in terms of the differential of negative/positive cognitive and emotional states in one of three general outputs: (a) nonphysical blocker (NPB) - the ill of the internal milieu outweighs the good; (b) nonphysical enhancer (NPE) — the good of the internal milieu outweighs the ill; and (c) nonphysical indifferent (NPI) - the good of the internal milieu is balanced with the ill.

Figure 3 a depicts an analytic perspective of the aforementioned states reflecting the resultant of the interaction of factors $\mathrm{A}$ and $\mathrm{N}$. The resultant may reside in one of four quadrants (left and right, top and bottom; each quadrant consists of four zones). Four possible states are evident for such interaction, namely: (a) preferred mode of operation in the presence of positive attributes of factors $\mathrm{A}$ and $\mathrm{N}$ $(\mathrm{A}+, \mathrm{N}+)$, that is, steady NPE (long-term zones in top-right quadrant in Figure 3a); (b) sub-optimal mode of operation in the unsteady state of the negative zone $(\mathrm{A}-, \mathrm{N}-)$, that is, unsteady NPB (top-right zone in bottom-left quadrant in Figure 3a); (c) transient $((\mathrm{A}-, \mathrm{N}+)$; $(\mathrm{A}+, \mathrm{N}-))$, that is, transient NPE or NPB (top-left and bottom-right quadrants), representing a changing state between steady and unsteady states; and (d) balance between factors $\mathrm{A}$ and $\mathrm{N}(|\mathrm{A}+| \sim|\mathrm{N}-|$; $|\mathrm{A}-| \sim|\mathrm{N}+|)$, that is, NPI.

The positive mode of operation (that is, $\mathrm{A}+, \mathrm{N}+$ ) allows one to work in a steady state by being more efficient and effective, and integrates the rational component of factor $\mathrm{A}$ and the positive impulse of factor N. It should be noted that the short-term steady state zone could not be sustained for a long period, with the person moving in and out of it to the long-term steady state operation. Alternatively, the suboptimal mode of operation emanates from the union of "low" negative thinking of factor A and the "low negative" state of factor $\mathrm{N}$ in the bottom-left quadrant of Figure 3a. The person in this zone is 
"vulnerable" to life and stress events and cannot sustain it for a long period of time. The person is also considered vulnerable in the remainder of this quadrant due to the impact of high A- or high N-and may be a threat for human health.

The transient states for NPB or NPE are situated in the top-left and bottom-right quadrants in Figure 3a. As depicted in the figure, one can only be transiently located in one zone, and possible shifts to neighboring zones may occur. To further clarify, factor $\mathrm{S}$ is capable, because of its one-to-many relationships, of additionally overcharging the negative intensity via a direct link to factor $\mathrm{K}$, hence changing its state occasionally depending on the interaction of factors A and N (Figure 3b). An example would be to instill a general state of intense "false" fear so as not to carry out the final decision decided upon by factor K. Another example is to generate an intense "false" state of overjoy, beyond the normal, which may influence the occurrence of future good decisions. This may occur when factor $\mathrm{S}$ observes that factor $\mathrm{K}$ is in a state of peace and tranquility; consequently it intends to change the internal milieu via false excitement by shifting the state of affairs into a high positive-energy source and a false, negative cognitive signal that can eventually turn into a high negative energy source.

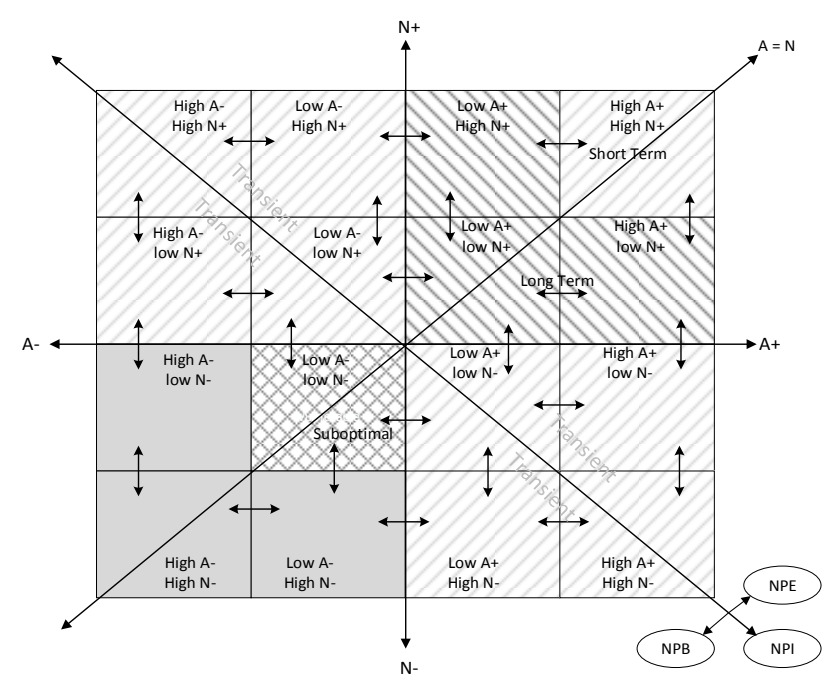

(a)

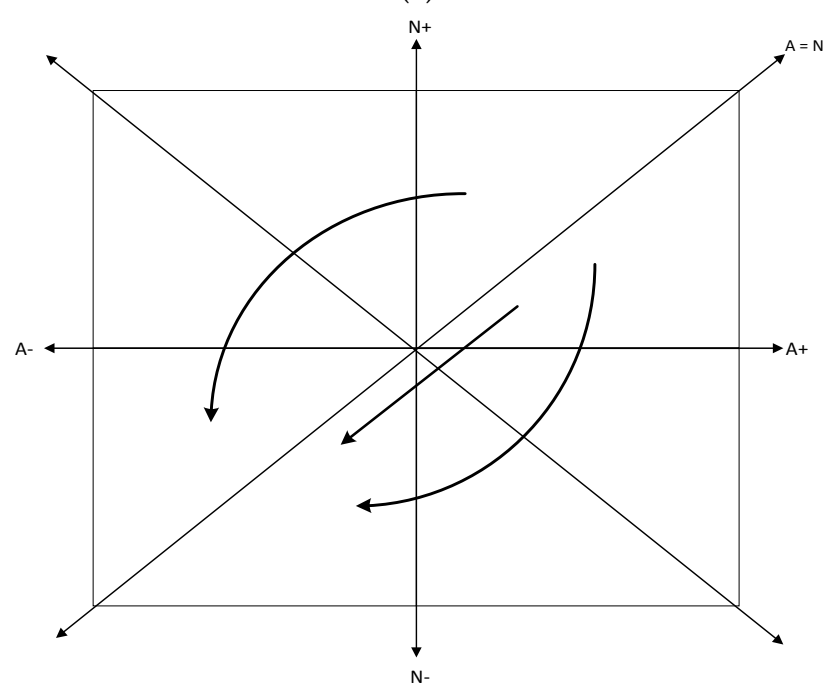

(b)

Figure 3. Analytic perspective of component interactions. (a) Interaction of factors A and N; (b) Influence of factor $\mathrm{S}$ on factor $\mathrm{K}$ (i.e., effect on state of factor $\mathrm{K}$ after the input of the resultant of factors $\mathrm{A}$ and $\mathrm{N}$ ). 
It should be noted that a comparison of Figures 2 and 3 illustrates the versatility of the analytic model. Each region of Figure 3 can be further divided into four smaller regions. This in turn provides a more precise version of a system state. In the same way each smaller region can be divided into four regions and so on. This division of the regions allows our model to be as discrete as needed for any given system. For example, for slowly-changing systems, a very discrete model can represent the system change in quantitative terms. Then if we know the timing of the change, we can immediately obtain the time rate of change or "system velocity". One can further observe that for passive systems, the natural tendency is to move down and to the left. That is, without growth and/or positive stimulation, the system will stagnate and move toward more negative configurations. Finally, with successive discretization following the pattern of Figure 3, we can develop a system rating according to position along and away from the lower left/upper right diagonal.

The aforementioned analytic formulation describing component interactions requires information-gathering from social agents designed to capture the different states of factors $\mathrm{A}$ and $\mathrm{N}$. This can be implemented in a multitude of ways via surveys or through the creation of focus groups in a social network environment. Surveys and focus groups are typically guided by scripts drawn from typical scenarios, leading to real-world challenges impacting individuals in their daily lives.

For example, individuals with multiple chronic conditions are confronted with the utilization of multiple medications thereby impacting their cognitive and emotional states and eventually resulting in non-adherence to medications [41]. The goal here is to change the behavior of these individuals in order to adhere to their medications to get well and eventually improve their physical, ecologic, and economic well-being. Therefore, the survey or focus group mission ought to be focused on the issues of interest to the members and the information gathering instrument should be designed in a way to extract the modes of operation of factors $\mathrm{A}$ and $\mathrm{N}$ together with their interactions with factors $\mathrm{S}$ and $\mathrm{M}$ with direct relevance to the situation at hand, as well as the inclusion of other any other factors deemed to be of importance, such as $\mathrm{A}^{\prime}$ and $\mathrm{N}^{\prime}$ from other individuals. Accordingly one can design smart logic to influence the persons in question.

It should be noted that one may be guided by analogy from the theory of stresses for different types of engineered materials. Although we are dealing with the intangibles of the aforementioned non-physical properties, we can however extract its projections on human behavior and action in exposure to various life events and accordingly develop a specialized instrument designed to record the challenges and opportunities to which humans are exposed. Accordingly, an algorithm can be built with inputs from these surveys to determine the inner states of the person and develop smart logic to better guide human actions to improve human, ecologic and economic well-being.

\section{Model Applications}

\subsection{Nonphysical Syndrome}

A subset of NPB may be referred to as nonphysical syndrome (NPS), representing acute and chronic maladaptive coping and traversing the interface into the physical domain (Figure 1b). It can present itself in the form of pathologic conditions impacting human health under the general rubric of maladaptive syndrome (MAS). It is hypothesized that the NPS phenomenon may lead to maladaptation for not only the person, but also for other entities in the larger system as a consequence of the person's actions potentially impacting ecosystem health and the natural environments. For example, at the individual level, a person may experience traumatic experiences during childhood, with the end result being a feedback from the physical domain to the non-physical domain as shown in Figure 1a. During the course of life events, the person may tune up or down his/her adaptive and maladaptive responses and MAS may transpire as a general pathologic condition leading to different chronic and acute conditions, with an end result of poorer health. In essence, MAS is a multi-system disease and usually affects a number of physiologic systems, organs and tissues during its course [42]. 
For further clarification, Figure 4 depicts the mapping of NPB and its elements from the non-physical domain into the maladaptive response and its constituents in the physical domain. There is usually a time lag between the NPB and maladaptive response. In addition, the NPS (clinical phase of NPB) maps onto the MAS (clinical phase of maladaptive response). In addition, MAS may consist of both medically-explained symptoms and medically-unexplained symptoms [43]. Thus, NPS contributes to the theory of disease in two ways. First, NPS can act together with the genetic component as modifiers for the random stochastic processes of bodily diseases. Second, NPS may contribute on its own, through non-random processes, as the main root cause of other bodily diseases.

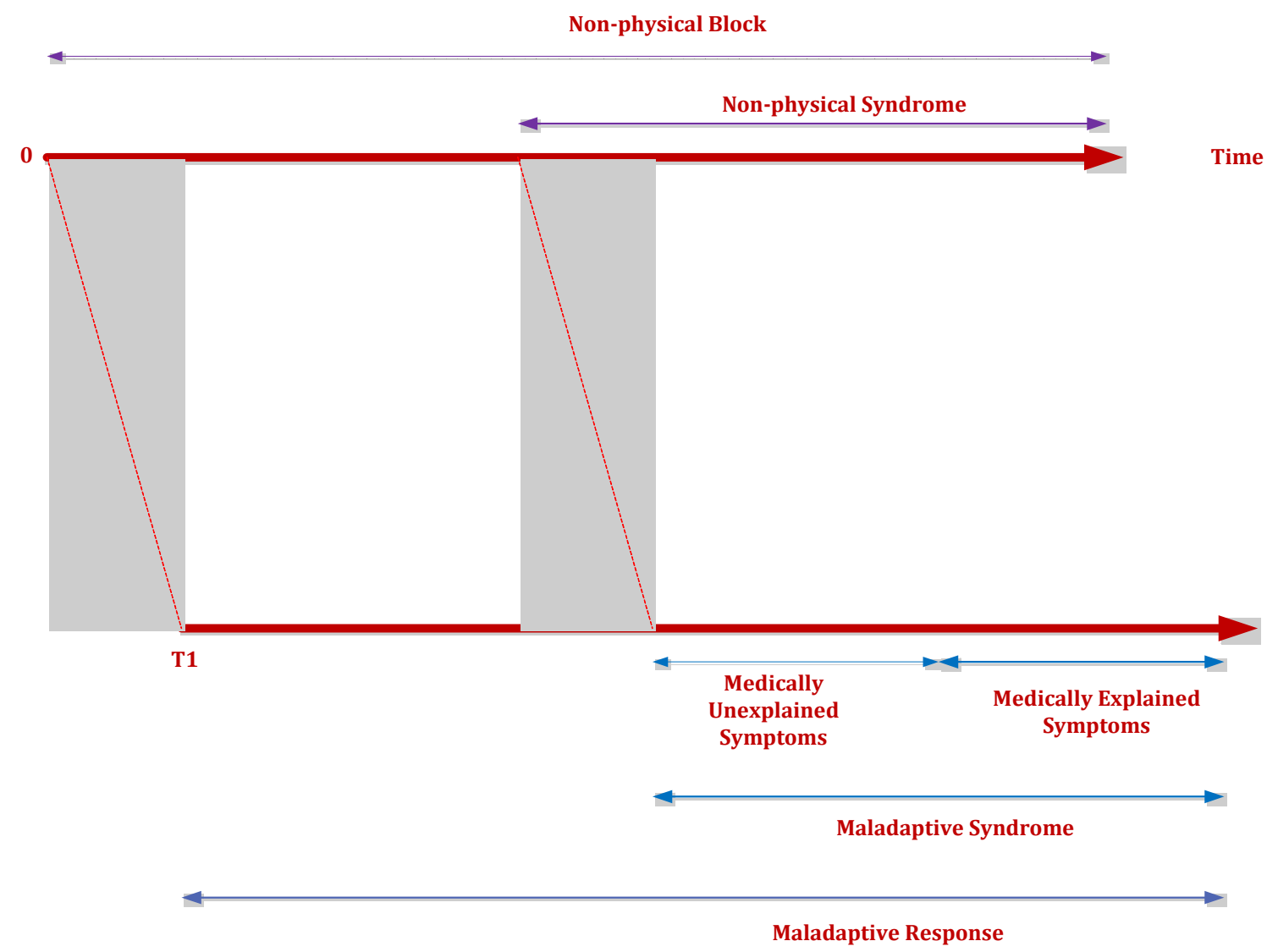

Figure 4. Mapping of non-physical block and syndrome into maladaptive response \& syndrome.

\subsection{Broadened Minds}

The concept of NPE can be instrumental as an integral part of developing value-added attributes for sustainable systems. It can be formulated in such tangible terms as the sustainability axiom of "Doing Good Is Good Business". This axiom can be used to advance the sustainability of the larger SEE complex adaptive system; consequently, it can be instituted as a device to prevent and mitigate the ills of system unsustainability. One must keep in mind that, although such an approach is the optimal path for sustainable systems, its time frame is intended for the longer term, making gains in incremental and sure ways, as opposed to non-optimal approaches which may result in significant gains in the short run, yet which may lead to long-term disastrous outcomes.

Within the context of this research, a "sustainability axiom" is a universal steady-state actionable plan with the intent of satisfying the needs and wants of all SEE stakeholders during an agreed-upon period of time, without creating conflicts and incompatibility among those needs and wants. "Doing Good and Not Doing Bad" Is "Good Business" is an example of one of the very few sustainability axioms available that may implement the spirit of the NPE. This axiom embodies a complex action plan into its meanings. 
1. "Doing Good" signifies the necessity of satisfying the needs and wants of all stakeholders without exception. As one can imagine, this axiom carries a long-term time span into it (as opposed to short fixes). It is not designed to be a disruptive action. Indeed, the action plan will consist of an inventory of solutions designed to incrementally and steadily improve system performance for all stakeholders at the same time. "Doing Good" enriches the SEE stakeholders by exploiting all potential positive energy and attributes of the system in a way that activates the "Non-physical Enhancers" for all social agents.

2. "Not Doing Bad" is the other side of the coin of "Doing Good", with the intent of preventing and filtering out bad and wicked actions from finding entry to the SEE system. It stipulates the criticality of avoiding conflicts and incompatibility among the SEE constituents. This component of the axiom is achieved via the control and minimization of the "Non-Physical Blockers" for all social agents representing the SEE system.

3. Either "Doing Good" or "Not Doing Bad" is a necessary condition but neither is individually sufficient on its own to induce the outcome of "Good Business". Both components are necessary and sufficient to produce the steady and incremental outcomes of "Good Business". This is essential both to activate and promote the system's adaptive properties and to deactivate and dampen the system's maladaptive properties.

As an example, the above strategy may provide a balancing act for sustainable emerging technologies such as engineered nanomaterials (ENMs) in the 21st Century, enabling a major boost to global economic activities, while preserving and enriching human, ecologic and economic well-being [19,44]. For ENMs to be sustainable across the SEE lifecycle trajectory, mental resources should be directed towards higher and diminished states of NPE and NPB, respectively, together with a balanced state of NPI, not impeding the harmonization of values among multiple stakeholders.

To illustrate this, it is common practice for venture capitalists in a start-up nanotechnology company to put extreme pressure on its nano-scientists and engineers to quickly design and manufacture ENM materials and nano-enabled products. This pressure, produced by the venture capitalists, acts as a feedback loop for the mental resources of the nano-scientists and the engineers. This tends to narrow the perspective of scientists and engineers, leading to system behavior and actions with a focus on fewer stakeholders in the SEE system such as emphasizing human needs at the expense of other biological species (e.g., nanomaterials may prove to be detrimental to other species along the particle lifecycle trajectory). As a result, the perspective of other constituents is not taken into account. This indicates that a broad, yet integrated, view is required whenever a product is manufactured for human use-particularly a consumer product. Moreover, the design of the product must be consistent with the SSE and its trajectory.

The NPE strategy is in line with the "broaden-and-build" theory of positive emotions which advocates that positive emotions broaden an individual's instantaneous thought-action reservoir (that is, interest sparks the urge to explore); and that by broadening an individual's thought-action reservoir, positive emotions promote the discovery of novel and creative actions, which in turn build the individual's personal resources ranging from physical and intellectual to social and psychological resources [45].

From the aforementioned discussion, one can deduce many corollaries that can assist in the production of a detailed actionable action plan with the most appropriate outcomes for the SEE system at hand. Currently, there is a gap in our knowledge with regard to the sustainability axioms and their derived corollaries with the intent to enhance the sustainability of SEE systems and to reduce vulnerabilities to sources of unsustainability. This area deserves future research with respect to three issues: (a) assessment of existing frameworks geared towards the sustainability of SEE systems; (b) deduction of sustainability axioms and their derived corollaries; and (c) demonstration of their applicability to case studies across the different domains of SEE to draw the lessons learned as a guide for further innovations within the sustainability sciences. 


\section{Discussion and Concluding Remarks}

Over the past several decades, there have been significant advances in science and technology and related trans-disciplinary fields of knowledge. However, the constituents of the larger SEE system are not achieving the expected improvements in different domains (e.g., human and ecologic health) consistent with these advancements nor with the related cost increases. This paradox may be understood in large part due to an absence of a model that adequately describes the root causes of sustainability/unsustainability (both positive and negative) embodied in the intrinsic and intangible nature of human and system behavior and action. Therefore, there are still gaps in knowledge that have not been elucidated.

These issues may be explained by a number of parameters with origin in social, environmental, technical, geographical, and political issues that result in unsustainable systems-systems that cannot keep pace with their basic needs, let alone the higher needs of human populations and other constituents of the larger SEE system. Other root causes may include, for example, a Western medicine perspective that has traditionally compartmentalized its view of health by separating not only mental from physical factors, but also by further segmenting individual people into "index diseases", rather than focusing on the whole person. This is due, in part, to the trend of medical "specialization" that has occurred over the past 50 years or so, resulting in an inadequate and counterproductive health-management approach.

It is our view that the above approach is going in a direction away from that of achieving optimum human, ecologic and economic outcomes, by focusing too much on technology and specialization as the answer for all ills. Therefore, this is limiting the ability to adequately improve the well-being of human populations and ecosystems in a long-term fashion. The work presented in this paper is foundational via its offerings of several thrusts aiming at enhancing and promoting the sustainability of SEE systems.

First, the PFA model implies that human outcomes and actions, regardless of geo-political circumstances, are determined by a number of influencers, including "non-physical", physical and social influencers, acting in a complex SOS to influence each person. Though the geo-socio-economic influencers can vary, it is felt that the pathway(s) to how these influencers impact individual outcomes and actions is universal for all mankind.

Second, an analytic formulation has been derived from the component interactions in the PFA architecture that explains the phenomena of a non-physical blocker (i.e., a system behaving in a negative mode), of an enhancer (i.e., a system behaving in a positive mode), and an indifferent (or neutral system) that are responsible for the adaptation and maladaptation of social agents, and accordingly for the sustainability and unsustainability of SEE systems. As pointed out, additional research is warranted to build instruments for information-gathering purposes from social agents and algorithms designed to capture the inner states of NPB, NPE and NPI with proxies from projections on human behavior, actions and outcomes. It should be kept in mind that the linguistic analytics presented in Figure 3a should not be construed as a linear system when projected at the digital level. Rather, it is a non-linear system with the linguistic processing as a device intended to manage the complexities of non-linear relationships at the digital level.

Third, examples were presented to illustrate the model applications at the individual level, such as non-physical and maladaptive syndromes. It has been hypothesized that NPB can contribute to the theory of diseases in two ways: (a) as a modifier for bodily medical conditions; and (b) as a root cause for non-random bodily disease processes. This line of work requires additional research with the exploratory intent of determining how to alter these responses with appropriate intervention strategies to improve human well-being. It is maintained that these interventions can divert an individual from, for example, poor health to good health if the imbalance of influencing factors leading to the negative patterns of MAS can be recognized in time early enough to impact its progression. Another important application is the utilization of the NPE phenomenon in the creation of value-added benefits for emerging technologies (e.g., ENMs) for all stakeholders of the larger SEE system. This step is essential 
at the predesign stage to ensure that adequate measures have been put in place to avoid disastrous consequences of large-scale technological artifacts. In such situations, a sociotechnical approach is required with customization for the application at hand.

In conclusion, a narrow consideration of what influences human outcomes and actions with a sole focus on technology will result in non-sustainable systems with negative outcomes impacting human populations and constituents of the larger SEE system. The model of "SOS" for human outcomes and actions, acknowledging both physical and non-physical influencers, can serve as a roadmap for defining and executing interventions that can induce positive change and minimize negative behavior for all stakeholders of society's many systems.

Acknowledgments: The work presented herein was based on the authors' initiatives and was not subject to external funding.

Author Contributions: A. Genaidy and R. Huston were responsible for conceiving the initial structural entities of the model and were validated by D. Dionysiou and W. Karwowski. The four authors contributed to all parts of the manuscript.

Conflicts of Interest: The authors declare no conflict of interest.

\section{Abbreviations}

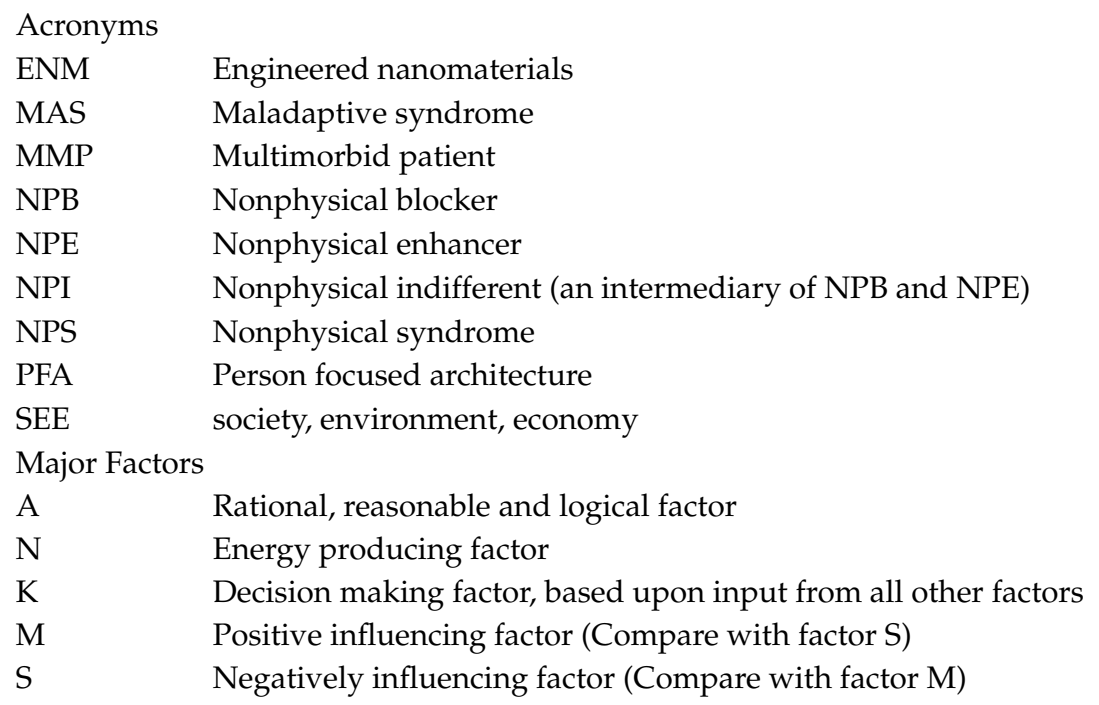

\section{References}

1. Fiksel, T.; Graedel, A.D.; Hecht, D.; Rejeski, D.; Sayer, G.S.; Senge, P.M.; Swackhamer, D.L.; Theis, L.T. EPA at 40: Bringing Environmental Protection into the 21st Century. Environ. Sci. Technol. 2009, 43, 8716-8720. [CrossRef] [PubMed]

2. Williams, P.R.D.; Dotson, G.S.; Maier, A. Cumulative Risk Assessment (CRA): Transforming the Way We Assess Health Risks. Environ. Sci. Technol. 2012, 46, 10868-10874. [CrossRef] [PubMed]

3. Depledge, M.H.; Stone, R.J.; Bird, W.J. Can Natural and Virtual Environments Be Used to Promote Improved Human Health and Wellbeing? Environ. Sci. Technol. 2011, 45, 4660-4665. [CrossRef] [PubMed]

4. Simon, H.A. A Behavioral Model of Rational Choice. Q. J. Econ. 1995, 69, 99-118. [CrossRef]

5. Simon, H.A. Rational Choice and the Structure of the Environment. Psychol. Rev. 1956, 63, 129-138. [CrossRef] [PubMed]

6. Simon, H.A. Motivational and Emotional Controls of Cognition. Psychol. Rev. 1967, 74, 29-39. [CrossRef] [PubMed]

7. Barabasi, A.L. The Origin of Bursts and Heavy Tails in Human Dynamics. Nature 2005, 435, $207-211$. [CrossRef] [PubMed]

8. Karwowski, W. A Review of Human Factors Challenges of Complex Adaptive Systems Discovering and Understanding Chaos in Human Performance. Hum. Factors 2012, 54, 983-995. [CrossRef] [PubMed] 
9. Pentland, A.; Liu, A. Modeling and Prediction of Human Behavior. Neural Comput. 1999, 11, $229-242$. [CrossRef] [PubMed]

10. Bonabeau, E. Agent-Based Modeling: Methods and Techniques for Simulating Human Systems. Proc. Natl. Acad. Sci. USA 2002, 99, 7280-7287. [CrossRef] [PubMed]

11. Homer, J.B.; Hirsch, G.B. System Dynamics Modeling for Public Health: Background and Opportunities. Am. J. Public Health 2006, 96, 452-458. [CrossRef] [PubMed]

12. Janssen, M.A.; Walker, B.H.; Langridge, J.; Abel, N. An Adaptive Agent Model for Analyzing Co-evolution of Management and Policies in a Complex Rangeland System. Ecol. Model. 2000, 131, 249-268. [CrossRef]

13. Jager, W.; Janssen, M.A.; de Vries, H.J.M.; de Greef, J.; Vlek, C.A.J. Behavior in Commons Dilemmas: Homo Economicus and Homo Psychologicus in an Ecological-Economic Model. Ecol. Econ. 2000, 35, 357-379. [CrossRef]

14. Bagozzi, R.P. Alternative Perspectives in Philosophy of Mind and Their Relationship to Structural Equation Models in Psychology. Psychol. Inq. 2011, 22, 88-99. [CrossRef]

15. Kusmartsev, F.V.; Kurten, K.E. Physics of the Mind: Opinion Dynamics and Decision Making Processes Based on a Binary Network Model. Int. J. Mod. Phys. B 2008, 22, 4482-4494. [CrossRef]

16. Pojola, C.; Stanculete, M.F. Biopsychosocial approach of gastrointestinal disorders. Clujul Med. 2014, 87, 95-97.

17. Kerr, C. Translating "Mind-In-Body": Two Models of Patient Experience Underlying a Randomized Controlled Trial of Qigong. Cult. Med. Psychiatry 2002, 26, 419-447. [CrossRef] [PubMed]

18. Iyer, I.; Albert, P.B.; Devi, S.M.A.; Devi, S.M.A.; Rakkiappan, C.N.; Mohanan, M.; Maharaj, D.O. The Science of Manifestations by God and Divinity leading to Inspiration and Persistence as Holy Books or Holy Sites or Evolved/Systematic Holy Ways of Life-A consequence of the Evolution of the Mind and the Heart inspired by Consciousness fields as explained in the New Consciousness model which scientifically converges Science and Spirituality with converged scientific and spiritual insights into the aspects of God, Soul, Karma, Birth and Re-birth, Machine Consciousness and Awareness, Plants, Animals, All Life, Natural and non-Natural forms and Convergence of Religious Teachings. Int. J. BioSci. Technol. 2013, 6, 37-73.

19. Tolaymat, T.; El Badawy, A.; Sequeira, R.; Genaidy, A. A System-of-Systems Approach as a Broad and Integrated Paradigm for Sustainable Engineered Nanomaterials. Sci. Total Environ. 2015, 511, 595-607. [CrossRef] [PubMed]

20. Shilling, C.; Mellor, P.A. Embodiment, Structuration Theory and Modernity: Mind/Body Dualism and the Repression of Sensuality. Body Soc. 1996, 2, 1-15. [CrossRef]

21. Sperry, R.W. Mind-Brain Interaction: Mentalism, Yes; Dualism, No. Neuroscience 1980, 5, 195-206. [CrossRef]

22. Borrell-Carrio, F.; Suchman, A.L.; Epstein, R.M. The Biopsychosocial Model 25 Years Later: Principles, Practice and Scientific Enquiry. Ann. Fam. Med. 2004, 2, 576-582. [CrossRef] [PubMed]

23. Alonso, Y. The Biopsychosocial Model in Medical Research: The Evolution of the Health Concept over the Last Two Decades. Patient Educ. Couns. 2004, 53, 239-244. [CrossRef]

24. Engel, G.L. The Need for a New Medical Model: A Challenge for Biomedicine. Science 1977, 196, 129-136. [CrossRef] [PubMed]

25. Brown, T.M. Cartesian Dualism and Psychosomatics. Psychosomatics 1989, 30, 322-331. [CrossRef]

26. Engel, G.L. How Much Longer Must Medicine's Science Be Bound by a Seventeenth Century World View? Psychother. Psychosom. 1992, 57, 3-16. [CrossRef] [PubMed]

27. Wade, D.T.; Halligan, P.W. Do Biomedical Models of Illness Make for Good Healthcare Systems? Br. Med. J. 2004, 329, 1398-1401. [CrossRef] [PubMed]

28. Wade, D. Complexity, Case-Mix and Rehabilitation: The Importance of a Holistic Model of Illness. Clin. Rehabil. 2011, 25, 387-395. [CrossRef] [PubMed]

29. Greene, R.A.; Dasso, E.; Ho, S.; Frank, J.; Scandrett, G.; Genaidy, A.M. Patterns and Expenditures of Multi-morbidity in an Insured Working Population in the United States: Insights for a Sustainable Health Care System and Building Healthier Lives. Popul. Health Manag. 2013, 16, 381-389. [CrossRef] [PubMed]

30. Greene, R.A.; Dasso, E.; Ho, S.; Genaidy, A.M. A Person-Focused Model of Care for the Twenty-First Century: A System-of-Systems Perspective. Popul. Health Manag. 2014, 17, 166-671. [CrossRef] [PubMed]

31. Genaidy, A.; Karwowski, W.; Shoaf, C. The Fundamentals of Work System Compatibility Theory: An Integrated Approach to Optimize Human Performance at Work. Theor. Issues Ergon. Sci. 2002, 3, 346-368. [CrossRef] 
32. Genaidy, A.; Salem, O.; Karwowski, W.; Paez, O.; Tuncel, S. The Work Compatibility Improvement Framework: An Integrated Perspective of the Human-At-Work System. Ergonomics 2007, 50, 3-25. [CrossRef] [PubMed]

33. Boardman, J.; Sauser, B. System of Systems-The meaning of of. In Proceedings of the IEEE International Conference on System of Systems Engineering, Los Angeles, CA, USA, 24-26 April 2006; pp. 118-123.

34. Gorod, A.; Sauser, B.; Boardman, J. System-of-Systems Engineering Management: A Review of Modern History and a Path Forward. IEEE Syst. J. 2008, 2, 484-499. [CrossRef]

35. DiMario, M.J.; Boardman, J.T.; Sauser, B.J. System of Systems Collaborative Formation. IEEE Syst. J. 2009, 3, 360-368. [CrossRef]

36. Dember, W.N.; Jenkins, J.J.; Teyler, T.J. The Group: Social Roles and Social Impact. In General Psychology, 2nd ed.; Wm. C. Brown: Dubuque, IA, USA, 1984; Chapter 18; pp. 731-739.

37. Dember, W.N.; Jenkins, J.J.; Teyler, T.J. The Group: Social Interaction. In General Psychology, 2nd ed.; Wm. C. Brown: Dubuque, IA, USA, 1984; Chapter 19; pp. 761-797.

38. Cohen, A. Heat, Stress, and Workload in an Era of Workplace Change, Ergonomics and Human Factors; Mark, L.S., Warm, J.S., Huston, R.L., Eds.; Springer: New York, NY, USA, 1987; pp. 155-169.

39. Hancock, P.A. Arousal theory, stress and performance: Problems of incorporating energetic aspects of behavior into human-machine systems function. In Ergonomics and Human Factors; Mark, L.S., Warm, J.S., Huston, R.L., Eds.; Springer: New York, NY, USA, 1987; pp. 170-179.

40. Seery, M.D. The Biopsychosocial Model of Challenge and Threat: Using the Heart to Measure the Mind. Soc. Personal. Psychol. Compass 2013, 7, 637-653. [CrossRef]

41. Starfield, B. Is patient care the same as person-focused care? Perm. J. 2011, 15, 63-69. [CrossRef] [PubMed]

42. Jakovljevic, M.; Ostojic, L. Comorbidity and Multimorbidity in Medicine Today: Challenges and Opportunities for Bringing Separated Branches of Medicine Closer to Each Other. Psychiatra Danub. 2013, 25, $18-28$.

43. Burton, C. (Ed.) ABC of Medically Unexplained Symptoms; Wiley-Blackwell: Oxford, UK, 2013.

44. Organization for Economic Co-operation and Development (OECD). Important Issues on Risk Assessment of Manufactured Nanomaterials; OECD: Paris, France, 2012.

45. Fredrickson, B.L. The role of positive emotions in positive psychology: The broaden-and-build theory of positive emotions. Am. Psychol. 2001, 56, 218. [CrossRef] [PubMed]

(C) 2017 by the authors. Licensee MDPI, Basel, Switzerland. This article is an open access article distributed under the terms and conditions of the Creative Commons Attribution (CC BY) license (http:/ / creativecommons.org/licenses/by/4.0/). 